\section{Epilepsie und Suizid: Gleiche Ursache?}

\begin{abstract}
Suizidraten von Patienten mit Epilepsie liegen bis zu zehnfach über den Raten der Allgemeinpopulation. An sich nichts Neues aber: US-Forscher können nun belegen, dass die Gefahr, sich selbst zu töten, bereits vor der Diagnose erhöht ist. Die Daten einer Kohortenstudie zeigen, dass das Risiko, einen ersten Versuch der Selbsttötung zu unternehmen, bei den künftigen Epilepsiepatienten 2,4-mal so hoch war wie bei den Kontrollen. Ein Folgeversuch war 1,8-mal so häufig. Die Forscher der Columbia University vermuten daher gemeinsame, bis dato noch unbekannte biologische Prozesse als Ursache.
\end{abstract}

Hesdorffer et al. JAMA Psychiatry 2016; 73:80-6

\section{Achtung Schilddrüse!}

Eine US-Studie enthüllt: Die Inzidenz von Schilddrüsentumoren steigt in Industrieländern stark an, vor allem bei jüngeren Frauen. Neben Brusttumoren wird sich das Schilddrüsenkarzinom in den nächsten fünf Jahren zu den am schnellsten wachsenden Krebserkrankungen entwickeln, so der Berufsverband Deutscher Nuklearmediziner (BDN). Die Prognose des Teams um Epidemiologin Hannah Weir entspreche dem Trend, der sich auch in Deutschland abzeichne. Der BDN registrierte 20134200 neu erkrankte Frauen, im Jahr 2000 waren es noch 2700 gewesen. „Trostpflaster" des BDN: In Deutschland werde nicht nur gut diagnostiziert, sondern auch gut therapiert.

Weir HKet al. Cancer 2015; 121(11):1827-37

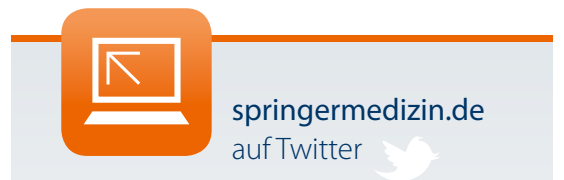

Auch im Winter heißt es

"Follower"

- twitter.com/springermedizin
"Lauschen" Sie unserem

Zwitschern und werden Sie zum

Höhere Rate schwerer Nebenwirkungen

\section{Knie-TEP bei Arthrose: Vor- und Nachteile}

In einer qualitativ hochwertigen Vergleichsstudie führte die Knie-TEP zu besseren Ergebnissen als konservative Maßnahmen allein.

Die Evidenz für den Nutzen der Knie-TEP untersuchten dänische Forscher um Søren T. Skou. Sie verglichen zwei Strategien: Die Op. plus konservative Maßnahmen, bzw. die konservativen Maßnahmen allein. Diese beinhalteten: 2-mal wöchentlich Training, eine Patientenberatung, Gewichtsreduktion, Einlagen sowie Analgetika. Die insgesamt 100 Teilnehmer wurden einer der beiden Gruppen zugelost. Nach zwölf Monaten wurde ausgewertet.

Beim primären Endpunkt, der Veränderung im „Knee Injury and Osteoarthritis
Outcome“-Score, lag die Op.-Gruppe klar vorn. Die TEP-Patienten schnitten bei den Kriterien Schmerzen, allgemeine Symptomatik, Alltagsaktivität und Lebensqualität signifikant besser ab.

Auf den entscheidenden Nachteil des chirurgischen Eingriffs weisen die Autoren besonders hin: die deutlich höhere Rate schwerer Nebenwirkungen, u. a. tiefe Beinvenenthrombose (3 Patienten), steifes Knie (3 Pat.) sowie tiefe Wundinfektion (1 Pat.).

Die Studie hat gezeigt, dass beide Strategien zu klinisch relevanten Verbesserungen führen, in $85 \%$ bzw. $68 \%$. Entscheidend ist es deshalb, das Für und Wider der Op. mit dem Patienten vorab abzuwägen.

Skou ST et al. NEJM 2015; DOI:10.1056/NEJMoa1505467
Primäre schlafgebundene Ursache

\section{Tasse Kaffee gegen den Kopfschmerz}

Wenn Kopfschmerz den Schlaf raubt, können die Ursachen vielfältig sein: Häufig stecken Atemprobleme dahinter, mitunter aber auch recht exotische Phänomene.

Schlafstörungen bei Kopfschmerzpatienten sind keine Seltenheit: Drei von vier Patienten finden keinen erholsamen Schlaf, erläuterte Prof. Dr. Svenja Happe von der Klinik für Neurologie in Telgte.

\section{Koffein als wirksame Prophylaxe}

Eine Besonderheit sind Kopfschmerzen, die ausschließlich im Schlaf auftreten. Die Neurologin nannte hier vor allem den primären schlafgebundenen Kopfschmerz, der die Betroffenen aus dem Schlaf reißt. Nach dem Aufwachen hält der Schmerz zwischen 15 min und bis zu $4 \mathrm{~h}$ an. Die Diagnosekriterien sind erfüllt, wenn über drei Monate hinweg mindestens zehn nächtliche Attacken im Monat auftreten und sich keine kranialen oder autonomen Ursachen finden lassen. Betroffen sind meist Ältere, zu zwei Dritteln Frauen, bei rund $60 \%$ treten die Schmerzen bilateral auf. Die Attacken beginnen in der Regel im REMSchlaf. Vermutet werden Veränderungen im suprachiasmatischen Kerngebiet.

Erstaunlicherweise scheint Koffein bei einigen Patienten gut prophylaktisch zu wirken: Mit einer Tasse Kaffee am Abend blieben viele die Nacht über schmerzfrei. Auch für NSAR, Lithium (300-600 mg), Indometacin (50-200 mg) sowie Flunarizin (5-10 mg) gebe es Berichte zur Wirksamkeit.

(mut) 